\title{
Demand Management of Station-Based Car Sharing System Based on Deep Learning Forecasting
}

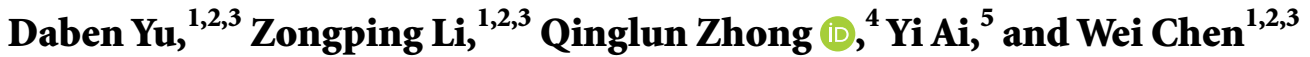 \\ ${ }^{1}$ School of Transportation and Logistics, Southwest Jiaotong University, 610031 Chengdu, China \\ ${ }^{2}$ National United Engineering Laboratory of Integrated and Intelligent Transportation, Southwest Jiaotong University, \\ 610031 Chengdu, China \\ ${ }^{3}$ Comprehensive Transportation Key Laboratory of Sichuan Province, Southwest Jiaotong University, 610031 Chengdu, China \\ ${ }^{4}$ Institut für Eisenbahnwesen und Verkehrssicherung, Technische Universität Braunschweig, Pockelsstr. 3, \\ 38106 Braunschweig, Germany \\ ${ }^{5}$ Civil Aviation Flight University of China, 618307 Guanghan, China \\ Correspondence should be addressed to Qinglun Zhong; q.zhong@tu-braunschweig.de
}

Received 24 April 2019; Revised 11 September 2019; Accepted 6 November 2019; Published 1 February 2020

Academic Editor: Gonçalo Homem de Almeida Correia

Copyright (c) 2020 Daben Yu et al. This is an open access article distributed under the Creative Commons Attribution License, which permits unrestricted use, distribution, and reproduction in any medium, provided the original work is properly cited.

\begin{abstract}
Metropolitan development has motivated car sharing into an attractive type of car leasing with the help of information technologies. In this paper, we propose a new approach based on deep learning techniques to assess the operation of a station-based car sharing system. First, we analyse the pick-up and drop-off operations of the station-based car sharing system, capturing the operational features of car sharing service and the behaviours of vehicle use from a temporal perspective. Then, we introduced an analytical system to detect the system operation concerning the spontaneous deviations derived from user demands from service provisions. We employed Long Short-Term Memory (LSTM) structure to forecast short-term future vehicle uses. An experimental case based on real-world data is reported to demonstrate the effectiveness of this approach. The results prove that the proposed structure generates high-quality predictions and the operation status derived from user demands.
\end{abstract}

\section{Introduction}

The recent development in motor drives and information technologies has promoted car sharing as a new solution to road transportation, due to its abilities to resolve the stressing conditions of congesting public roads and insufficient parking spaces [1]. Car sharing services can allow more access to vehicles without acquiring as many ownerships as private ones. In most densely populated large cities, its charm can help improve metropolitan traffic conditions from excessive car ownership [2]. In 2016, China's Internet car sharing market expanded considerably, including new service providers such as G. In August 2017, the Ministry of Communications and the Ministry of Housing and Urban-Rural Development of China jointly issued the "Guiding Opinions on Promoting the Healthy Development of Small and Minibus Leases" [3], encouraging new energy vehicles to be leased on time.

Within the wide ranges of public transport systems, the practical options for a metropolis like Chengdu are rather limited in terms of resolving its traffic dilemmas. Automobile holdings in Chengdu amount to more than 4.5 million by the end of 2017 [4], and most of them reside within its urban districts, occupying parking spaces and jamming public roads. Free-floating car sharing system provides shared car access, but free-floating cars are hard to manage and can cause the same issue of disorderly parking as private cars do. In the meantime, a station-based car sharing system establishes its business with its own parking spaces and can become advantageous in cities where parking can be a real headache. In the meantime, considering the requirements on urban environment protection and $\mathrm{CO}_{2}$ emission reduction, electric car sharing systems can serve as a viable choice, providing better urban transport service that allows more privacy $[5,6]$.

The further expansion of car sharing business will intensify the competition until the market reaches a phase of integration [7]. Thus, for a car sharing company to operate its business properly and to secure profitability, the temporal distribution of demands in different stations and mechanisms for 
inner-system operations should be identified before relevant marketing strategies can be made. Therein, user preferences are most critical in terms of vehicle uses and the level of service (LOS) [8].

We have learned from the market that car sharing is still in a development trend, and the fundamental problem of car sharing profitability is related to the demand side. Currently, the effective utilization rate of shared cars is quite low and fails to reach the threshold required for profit. Then, improving operational efficiency is the key point to enterprise profitability. Therefore, the car sharing enterprises are in urgent need of a clear business model and operating model, so that to get rid of high costs.

This paper considers the demand management of station-based car sharing service systems, whose customers are charged for the time used. The operation of car sharing systems can be analysed based on the usage of vehicles in the system, which we formulate as a discrete event model. And better operation of the car sharing business requires improved prediction of vehicle demands. Therefore, we wish to predict car sharing demand using state-of-the-art deep learning approaches. Thus, this paper attempts to apply deep learning forecasting techniques in the marketing analysis of station-based car sharing service. The contributions of this paper are as follows: (1) a discrete event model is established to help perceive the vehicle usage behaviours for the car sharing system; (2) a Long Short-Term Memory (LSTM) structure is formulated to forecast station-based car sharing demand; (3) the results from LSTM prediction are analysed using the discrete event model.

The rest of this paper is structured as follows. Section 2 reviews past works and existing methods in the fields of car sharing and forecasting. Section 3 examines the interaction between car sharing service providers and users and analyzes the operation status of the car sharing system. In alignment with the forecasting car sharing demands, the LSTM structure used in this article is given a detailed description in Section 4. Section 5 reports a case study on forecasting and analysing real-world car sharing demands in Chengdu. Section 6 concludes this work briefly and presents an outlook.

\section{Literature Review}

One of the earliest car sharing initiatives, "Sefage" (Selbstfahrergenossenschaft), operated in Zurich, Switzerland, from 1948 to 1998 [1,9-11]. The car sharing market in China started in 2010. More than 40 car sharing companies together operate more than 1,030,000 vehicles in China by Nov. 2018 [12]. Car sharing operators have developed several models, including rebate, interest-free instalment, and P2P (peer-topeer) $[13,14]$, and are expecting a growth of more than 50 percent.

Existing literature has explored the environmental and socio-economic impact of car sharing services. Prettenthaler and Steininger [15] discovered that private car ownership had shifted to usage, and this shift was often promoted in the concept of sustainability. Their analysis showed that policy tools must directly promote car service used instead of merely changing its marginal economic costs. Fellows and Pitfield [16] used cost-benefit analysis techniques to demonstrate that car sharing can bring net benefits comparable to the revenue generated by the main road plan. After an overview of the history of car sharing in Europe and North America, Katzev [17] reviewed the history of car sharing in Europe and North America and concluded three findings: the correlation of car sharing use to reduction of financial expenses, the most influential factor of car sharing use, and private car ownership. Firnkorn and Müller [18] assessed the environmental impact of car sharing services using data from Car2go, featuring the average $\mathrm{CO}_{2}$ emission reduction per user. Car sharing, as a complementary mode of transportation, is becoming more and more important, especially in metropolitan areas. This means that further development of planning tools is needed to consider this "new mode" in the planning process. An agent-based travel demand model that simulated the travel behaviour of the population in the greater Stuttgart area for one week achieved the integration of car sharing. And the model permitted longitudinal analysis of the intensity and variability of car sharing usage [19].

Research on the forecasting of car sharing system demand is plentiful. In terms of nonAI prediction methods, Wang et al. [20] proposed a new method to forecast and relocate car sharing service vehicles based on an inventory management model consisting of three main components: focus forecasting, inventory replenishment, and microscopic traffic simulation. Ciari et al. [21] introduced activity-based micro-simulation for estimating demands of car sharing, and they sought to develop appropriate evaluation tools for innovative transport modes. Yoon et al. [22] explored Beijing's potential car sharing demands, fleet size, and economic performance. Car sharing mode split is estimated by a stated preference choice modelling exercise. Adequate fleet size is estimated through a Monte Carlo simulation that includes factors such as vehicle types (electric or gasoline vehicle), charger types for electric vehicles (level 2 or level 3 chargers) that influence charging time, arrival rates, travel distance, and travel time based on the time intervals (peak or nonpeak hours). Alfian et al. [23] proposed forecasting relocation to solve car distribution imbalances for one-way car sharing services. Real case data sets have been used to find the best simulation results. As a result, the impact of predictive relocation on high system utilization and reservation acceptance ratio can be seen compared to traditional relocation methods.

In terms of AI prediction methods, in the past three years, more and more DL algorithms have been utilized in sharedbased vehicles mode prediction, such as taxi, express, operating lease, and car sharing, due to their ability to capture complex relationships from large amounts of data. Ghaderi et al. [24] employed Recurrent Neural Networks (RNNs) to forecast the short-term spatio-temporal wind speed, and they modelled spatio-temporal information based on a graph, representing data generating entities with nodes and their interactions with edges. The results showed significant improvement compared with a set of benchmarks models. Furthermore, Epelbaum et al. [25] explored time-dependent data forecasting using deep learning frameworks, such as FNN, CNN, and LSTM. $\mathrm{Xu}$ and Lim [26] proposed an evolutionary neural network for predicting the net flow of a car sharing system. Despite the 
disadvantages in the prediction process, ENN has shown excellent performance compared with classical time-series predictions. Khoo [27] used a neural network as a simulation model to predict the demands at stations in a car sharing system. This research introduced intelligent filtering technology, including outlier analysis and cluster analysis, to remove noises from the feeding data. The results showed that outlier analysis could better improve the accuracy of the predictions compared with cluster analysis. Yu et al. [28] proposed a space-time graphic convolutional network (STGCN) for time series traffic prediction. The study achieved "faster training, easier convergences, and fewer parameters with flexibility and scalability". To make full use of the temporal and spatial characteristics of traffic data. Zhang et al. [29] proposed a short-term traffic flow prediction model based on the Convolutional Neural Network (CNN) deep learning framework. In the proposed framework, the optimal input data time lags and the amount of spatial data are determined by the spatiotemporal feature selection algorithm (STFSA), and the selected spatiotemporal traffic flow features are extracted from the actual data and converted into a twodimensional matrix. Then, we used CNN to learn these features and built a predictive model. It was also verified that the proposed method is superior to the baseline model in terms of accuracy. Ai et al. [30] employed a deep learning approach, named the convolutional long short-term memory network (Conv-LSTM), to address the spatial dependences and temporal dependences. The spatiotemporal variables including the number of bicycles in area, distribution uniformity, usage distribution, and time of day as a spatiotemporal sequence in which both the input and the prediction target are spatiotemporal 3D tensors within one end-to-end learning architecture. Experiments show that conv-LSTM outperforms LSTM on capturing spatiotemporal correlations. Wang et al. [31] proposed a Global Positioning System (GPS)-data-driven approach to solving car sharing system demand forecasting problems. Historical vehicle GPS data can match the user's current trajectory and infer its possible destination. A four-step procedure was proposed based on GPS trajectory similarity measurements. The method also considered station correlations and historical user destinations. A case study proves the feasibility of the dynamic prediction program of the method. Ke et al. [32] proposed a new deep learning method, convolutional long-term memory network (FCL-Net), which includes spatial, temporal, and exogenous dependencies in an end-to-end learning architecture. The results showed that the proposed approach outperforms the best benchmark $\mathrm{CNN}$, and custom random forest reduced the training time significantly with a slight accuracy loss. To bridge the short-term forecast of a supplydemand gap for online car-hailing services, $\mathrm{Gu}$ et al. [33] proposed a novel spatio-temporal deep learning model (S-TDL). The model is composed of three sub-models: spatiotemporal variable model, spatial attribute variable model, and environment variable model. It can capture the impact of spatio-temporal correlation, regional difference and environmental change on the supply-demand gap. Moreover, a feature selection method named feature clustering-maximum information coefficient two-stage feature selection is proposed to screen out the important features which are strongly correlated with the supply-demand gap, improve training efficiency. Two deep learning architectures leverage word embeddings, convolutional layers and attention mechanisms for combining text information with time-series data have been proposed [34]. Using publicly available taxi data from New York, it empirically showed that by fusing these two complementary cross-modal sources of information, the proposed models could significantly reduce the error in the forecasts.

To reduce the operating cost of the car sharing company and improve users' satisfaction, a one-way car sharing system optimization model considering the users' time window requirements is constructed with the minimum total cost (including scheduling cost and fines cost). Then, an improved tabu search particle swarm optimization algorithm is proposed to solve the model [35]. On the other hand, to make daily booking services improve user satisfaction, and to provide operators with additional information about demand patterns. Molnar and Correia [36] proposed an innovative appointment execution technique. It can keep the system profitable and achieve high service quality while achieving a longer settling time.

In conclusion, existing literature has shown the evolvement of forecasting methodologies and their applications in related fields, which require either short- or long-term predictions, such as wind speed [24] and free-floating bike distribution [30]. However, it is not difficult to see that most of the researchers are directly optimizing the management of shared vehicle systems from the perspective of demand. Ignoring the most fundamental crux of finding out the difficulty of trip sharing is the most effective way to cure the problem. Therefore, this paper focused on the demand management of car sharing. For the system operation, we wish to find out the attractiveness of car sharing services to driving time. We also wish to clarify the difference between the market demands and the servicing vehicles in different stations and time periods through evaluating the operation of the existing car sharing system. In terms of methodology, we attempt to combine deep learning techniques with car sharing operation modelling so that the predicted data can be directly involved in detecting the deviations of servicing vehicles from market demands caused by system operation.

\section{Status of System Operation}

To tackle the operation status for car sharing systems, we intend to investigate the interactions between car sharing service providers and users, namely pick-up and drop-off. And we detect the deviation of vehicle distribution from customer demand by calculating the spontaneous operation process.

3.1. Assumptions. The current car sharing companies usually provide their service on mobile platforms, and users reserve vehicles from their smartphones. Car sharing companies usually allow a short duration of time for vehicle pick-up after reservation, since it is not efficient for on-demand ride sharing systems-which are supposed to circulate fast and 
easy-to allow long-duration pick-up. To analyze the vehicle pick-up and drop-off of the system, we make the following assumptions:

\subsubsection{Historical Data Represent the Real Trip Intentions of the}

Users. We assume that all users of car sharing service make rational decisions based on their trip intentions. Therefore, the trip demand has a rather reliable consistency under certain conditions (such as price rate and traffic condition). This allows us to show the systemic status of the operation under constant price and traffic conditions. In this study, vehicle usage pricing is constant.

\subsubsection{The Perturbation of Urban Traffic does not Affect the Daily}

Operation of Car Sharing Operations. Though the real-time traffic condition impacts the routing choices for vehicles en route, the drivers are aware of the traffic condition in which they are involved and make their trips. Therefore, the specific urban traffic condition can be disregarded.

\subsubsection{Vehicles are Uninterruptedly Made Available to}

Users. With either gasoline- or electric-powered vehicles, we assume that car sharing companies have certain maintenance procedures that allow the vehicles to circulate efficiently and satisfy customer demand without interrupt. Therefore, the model does not incorporate the repair or the refueling (or charging) procedures.

\subsection{Analysis of Vehicle Pick-Up and Drop-Off}

3.2.1. Operator Perspective. The operation of the car sharing station can be approached as discrete-time events [37]. Let $\tau$ denote the time when an action (a vehicle pick-up or drop-off) at an arbitrary station in the system. Let $(\tau, \tau+1)$ denote a time fraction, during which no action occurs between time $\tau$ and $\tau+1$. The number of vehicles at time fraction $(\tau, \tau+1)$ in station $i$ can be denoted as $s_{i}^{\tau, \tau+1}$, where $i \in\{1, \ldots, I\}$ and $I$ denotes the total number of stations in the system. And the number of vehicles in use at time fraction $(\tau, \tau+1)$ can be denoted as $r^{\tau, \tau+1}$, where $(\tau, \tau+1)$ represents the time fraction between two actions in the system within the time span (usually one day). We define virtual station $I+1$ to include those vehicles that are in operation. Term $r^{\tau, \tau+1}$ can be replaced by the number of vehicles in station $I+1$ at time fraction $(\tau, \tau+1) s_{I+1}^{\tau, \tau+1}$, which can be used for expressing the operation state of the whole system at time fraction $(\tau, \tau+1)$ when combined with $s_{i}^{\tau_{g}, \tau_{g+1}}$. At time fraction $(\tau, \tau+1)$, we have $\sum_{i=1}^{I+1} s_{i}^{\tau, \tau+1} \leq C_{0}$ where $C_{0}$ denotes the total number of vehicles in the system. Thus, the status of the system during time fraction $(\tau, \tau+1)$ can be expressed as

$$
S^{\tau, \tau+1}=\left\{s_{1}^{\tau, \tau+1}, \ldots, s_{I}^{\tau, \tau+1}, \ldots, s_{I}^{\tau, \tau+1}, s_{I+1}^{\tau, \tau+1}\right\}
$$

where $S^{\tau, \tau+1}$ denotes the operation status of the car sharing system at time fraction $(\tau, \tau+1)$.

Notice that $\tau$ indicates the time when an action occurs in the system, not a specific station at time $\tau$, we notice that the status of stations drives the operation status, and the number of remaining vehicles at station $i$ at time fraction $(\tau, \tau+1)$, $s_{i}^{\tau, \tau+1}$, usually holds much longer than the duration of time fraction $(\tau, \tau+1)$. Let $\tau_{i}$ denote the action when a vehicle is picked up or dropped off at station $i$ in the system, and $\tau_{i}=\tau$ when an action occurs at station $i$ in the system. Therefore, the number of vehicles at station $i$ during time interval $\left[\tau_{i}, \tau_{i}+1\right)$ can be expressed by $s_{i} \tau_{i}, \tau_{i}+1$.

Denote a vehicle pick-up from station $i$ at time $\tau_{i}$ as $p_{i}^{\tau}$, while a vehicle drop-off at station $i$ at time $\tau_{i}$ as $d_{i}^{\tau}$. We regard all freshly dropped-off vehicles are available to users for operation.

When a vehicle is picked up from station $i$ at time $\tau_{i}$,

$$
s_{i}^{\tau_{i}, \tau_{i}+1}-s_{i}^{\tau_{i}-1, \tau_{i}}=p_{i}^{\tau_{i}}=-1 .
$$

When a vehicle is dropped off to station $i$ at time $\tau_{i}$,

$$
s_{i}^{\tau_{i}, \tau_{i}+1}-s_{i}^{\tau_{i}-1, \tau_{i}}=d_{i}^{\tau_{i}}=1 .
$$

Let $\left(t_{j}, t_{j+1}\right)$, where $j \in\{1, \ldots, J\}$, denote the $j$-th time slice in one operation day, where $t_{j}$ and $t_{j+1}$ denote the start and end of the time slice. Consider the operation of the system during time slice $\left(t_{j}, t_{j+1}\right)$, which includes many instances of time fractions. The total car sharing demands originating from station $i$ can be expressed by the vehicles picked up from station $i$ during time slice $\left(t_{j}, t_{j+1}\right)$, denoted as $A_{i}^{t_{j}, t_{j+1}}$, which can be given by

$$
A_{i}^{t_{j}, t_{j+1}}=\sum_{\tau \in\left(t_{j}, t_{j+1}\right)} p_{i}^{\tau} .
$$

In the same vein, the total demands terminating at station $i$ during time slice $\left(t_{j}, t_{j+1}\right)$ be $E_{i}^{t_{j}, t_{j+1}}$, given by

$$
E_{i}^{t_{j}, t_{j+1}}=\sum_{\tau \in\left(t_{j}, t_{j+1}\right)} d_{i}^{\tau} .
$$

3.2.2. User Perspective. The utilization of ride sharing vehicles can be either based on short-term reservation, or ad-hoc, which can be generalized as an aggregated process, as illustrated in Figure 1.

A registered customer of the system books his or her use of a vehicle in the system, to complete the wanted journey via a route from his origin to his desired destination, with resorting to a pick-up and drop-off station of the car sharing system.

From the perspective of car sharing system operation, the main content of system dispatch, be it through algorithmic matching or human intervention, is to deal with car booking requests on the system made by its users. This process can be generalized as customer aggregation by authorizing them the access of currently available vehicles in the system according to their booking specifications, including the distance to be traversed during the trip.

The transport demands on the car sharing system can be described in the form of an O-D matrix based on traffic theory, and each element of this matrix includes a complete record of ride sharing vehicle usage. A complete record of successful 


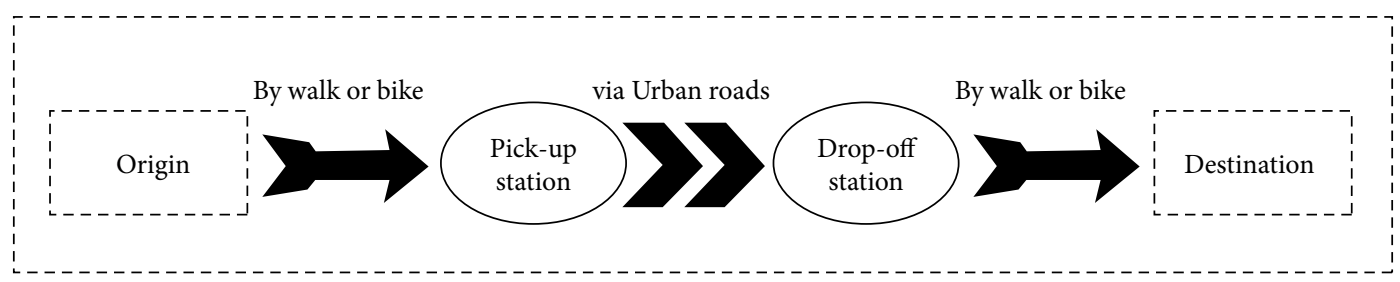

FIgURE 1: Basic process.

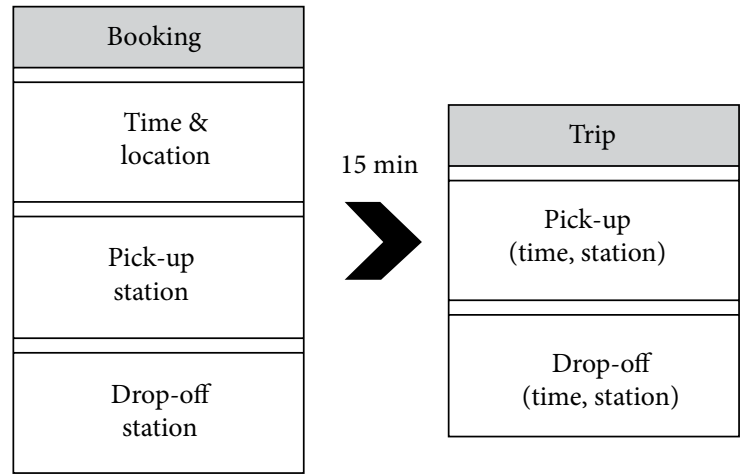

Figure 2: A record of a successful car sharing trip.

car sharing trips should assume such form as illustrated in Figure 2.

The time and location of actual trips can be collected and analysed. Pick-up and drop-off information can be easily obtained from the system log, and they can be paired up with the number of e-cars used by the users. Thus the operation status of the system relating to car sharing demand can be approached [38, 39].

Suppose that a vehicle was picked up from station $i$ at time $\tau_{i}$, and dropped off at station $k$ at $\tau_{k}$. The driving time $d_{v}$ for the vehicle can be given by

$$
d_{v}=\tau_{k}-\tau_{i}
$$

3.3. Spontaneous Deviation. Due to customer demands of diverse origin-and-destinations, the distribution of vehicles within car sharing stations spontaneously changes during operation of station-based car sharing systems. We define the result of vehicle pick-up and drop-off as spontaneous deviation, which can be regarded as the comprehensive indicator for system operation under the configurations of pricing, vehicle distribution, and demand distribution. Spontaneous redistribution of vehicles is most important managing subject for both free-floating and station-based car sharing as it impacts the service capacity of the system by lowering the access of vehicles for the users. Thus, various sorts of business decisions concerning the relocation of car sharing resources revolve around it. We present a mathematical evaluation of spontaneous vehicle deviation in station-based car sharing system in this section.

The cumulative result of car pick-ups and drop-offs in the station $i$ during time slice $\left(t_{j}, t_{j+1}\right)$ can be calculated by

$$
z_{i}^{t_{j}, t_{j+1}}=A_{i}^{t_{j}, t_{j+1}}+E_{i}^{t_{j}, t_{j+1}}
$$

Define $u^{t_{k}, t_{k+1}}=\left(z_{1}^{t_{k}, t_{k+1}}, \ldots, z_{i}^{t_{k}, t_{k+1}}, \ldots, z_{I}^{t_{k}, t_{k+1}}, z_{I+1}^{t_{k}, t_{k+1}}\right)^{\prime}$ to show the deviation of vehicle distribution of all stations in the car sharing system during time slice $\left(t_{j}, t_{j+1}\right)$. Define $u_{i}=\left(z_{i}^{t_{1}, t_{2}}, \ldots, z_{i}^{t_{j}, t_{j+1}}, \ldots, z_{i}^{t_{n-1}, t_{n}}\right)$ to show the deviation of vehicle distribution of different time slices at the station $i$. Define a matrix $Z=\left(z_{i}^{t_{j}, t_{j+1}}\right)_{(I+1) \times(n-1)}$, where $j \in\{1, \ldots, J\}$, and $i \in(1, \ldots, I, I+1)$, to show the deviation created during system operation. Let $Z=\left(u^{t_{1}, t_{2}}, \ldots, u^{t_{j}, t_{j+1}}, \ldots, u^{t_{n-1}, t_{n}}\right)=$ $\left(u_{1}, \ldots, u_{i}, \ldots, u_{I}, u_{I+1}\right)^{T}$. Matrix $Z$, therefore, represents the balances of the vehicles in the system over the concerned period, depending on the purpose of the study (usually being one operation day). For $i \in\{1, \ldots, I, I+1\}$ and $j \in\{1, \ldots, J\}$, the matrix can be written as

$$
Z=\left[\begin{array}{ccccc}
z_{1}^{t_{1}, t_{2}} & \ldots & z_{1}^{t_{j}, t_{j+1}} & \ldots & z_{1}^{t_{J-1}, t_{J}} \\
\vdots & \ddots & \vdots & . \cdot & \vdots \\
z_{i}^{t_{1}, t_{2}} & \ldots & z_{i}^{t_{j}, t_{j+1}} & \ldots & z_{i}^{t_{J-1}, t_{J}} \\
\vdots & . \cdot & \vdots & \ddots & \vdots \\
z_{I}^{t_{1}, t_{2}} & & z_{I}^{t_{j}, t_{j+1}} & & z_{I}^{t_{J-1}, t_{J}} \\
z_{I+1}^{t_{1}, t_{2}} & \cdots & z_{I+1}^{t_{j}, t_{j+1}} & \cdots & z_{I+1}^{t_{J-1}, t_{J}}
\end{array}\right] .
$$

Based on matrix $Z$, the following statistical terms can be calculated

$$
\begin{gathered}
I^{t_{j}, t_{j+1}}=\sum_{i=1}^{I}\left(z_{i}^{t_{j}, t_{j+1}}\right), \\
I_{i}=\sum_{j=1}^{J-1}\left(z_{i}^{t_{j}, t_{j+1}}\right), \\
I=\sum_{i=1}^{I} \sum_{j=1}^{J-1}\left(z_{i}^{t_{j}, t_{j+1}}\right), \\
D^{t_{j}, t_{j+1}}=\frac{\sum_{i=1}^{I}\left[\left(z_{i}^{t_{j}, t_{j+1}}\right)^{2}\right]}{I}, \\
D_{i}=\frac{\sum_{j=1}^{J-1}\left[\left(z_{i}^{t_{j}, t_{j+1}}\right)^{2}\right]}{J-1}, \\
D_{i}=\frac{\sum_{j=1}^{n-1}\left[\left(z_{i}^{t_{j}, t_{j+1}}\right)^{2}\right]}{n-1} .
\end{gathered}
$$

Equation (9) gives the aggregated trip intentions of customers using the car sharing system during time slice $\left(t_{j}, t_{j+1}\right)$; Equation (10) gives the aggregated trip intentions of customers using car sharing station $i$ over the concerned period; Equation (11) gives the intention of customers using car sharing services 
overnight; Equation (12) gives the deviation of customers' intentions from ideal operation condition of the system during time slice $\left(t_{j}, t_{j+1}\right)$, with vector $I^{t_{j}, t_{j+1}}$ fully consisted of zeros as the ideal operation condition; Equation (13) gives the deviation of customers' intentions from ideal operation of car sharing station $i$ over the concerned period, with vector $I_{i}$ fully consisted of zeros as the ideal operation condition; Equation (14) gives a rough estimation of the deviation of customers' intentions from ideal operation, with matrix $Z$ fully consisted of zeros as the most ideal condition.

\section{An LSTM Structure for Station-Based Car Sharing Demand Forecasting}

Deep learning has been considered as a mature application technology in various fields for a long time, including traffic management and time series prediction. Long Short-Term Memory (LSTM) solves many time-series problems that cannot be solved by a feedforward network using fixed-size time windows. This section proposes a deep learning based on the LSTM structure for short-term prediction of time series requirements for a station-based car sharing system.

\subsection{Temporal Features of Station-Based Car Sharing De-} mand. The prediction of car sharing system is essentially a time series forecasting problem. However, the imbalanced distribution of vehicles and demands will have a large impact on its time and space evolution. So, it can be a multidimensional space-time prediction problem. Therefore, we only consider the time series prediction problem in this paper. In the long-term research, it will still be a multidimensional space-time prediction problem. We define the time variables as follows:

4.1.1. Pick-up/drop-off. Real-time system forecasting is always difficult, but car sharing system analysis on a larger scale does not require real-time information, such as time fragments between pick-up/drop-off. To achieve the managerial purpose of the analysis prescribed previously in this article, numbers of vehicle pick-up $A_{i}^{t_{j}, t_{j+1}}$ and drop-off $E_{i}^{t_{j}, t_{j+1}}$ are defined one of time variables.

4.1.2. Arbitrary time. Commuting and noncommuting variants are incorporated to decipher the temporal features of station-based car sharing demands from operation data. Since car sharing is one of many business modes within the range of urban transport services, it is, therefore, subject to the urban traffic patterns. Morning and evening peaks, defined by urban traffic patterns, shape the urban traffic pattern on a normal working day in general. Trip attributes during workdays also significantly diverse, featuring mostly leisure activities and few commutes. And there are almost all leisure trips and no commutes during holidays. With that said, the trip pattern using car sharing services can be related to the urban traffic patterns, though not necessarily identical, which requires exploration. Let $f_{s}$ sign the time during a day (time-of-day), and an arbitrary time can be divided based on two urban traffic peaks as $f_{s}$ :

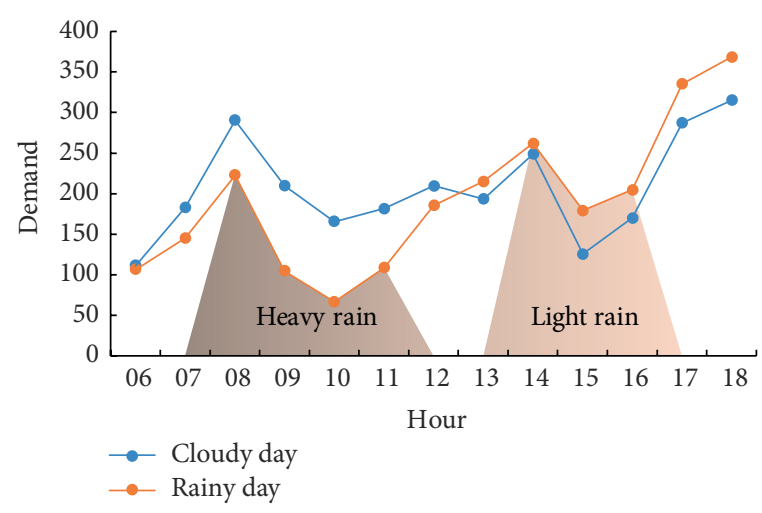

Figure 3: Comparison of different weather conditions for two identical weekdays.

(1) $f_{s}=1$ if the time is in the inactive period before the morning peak;

(2) $f_{s}=2$ if the time is during morning peak;

(3) $f_{s}=3$ if the time is between morning and evening peak;

(4) $f_{s}=4$ if the time is during evening peak;

(5) $f_{s}=5$ if the time is after the evening peak on the same day.

Let $y_{s}$ sign a day (day-of-week), and an arbitrary day can be classified as:

(1) $y_{s}=1$ if the day is a working day;

(2) $y_{s}=0$ if the day is a nonworking day.

4.1.3. Weather. Weather is also an important factor influencing the utilization of car sharing services in choosing the desired means of transport. We use identical weekdays but the different weather to compare the two-day demands. As shown in Figure 3, the cloudy day (northwest wind, breeze) has a temperature of $23-26^{\circ} \mathrm{C}$. Rainy day (southeast wind, level 1) has a temperature of $14-18^{\circ} \mathrm{C}$. Heavy rains between 6:30 and 11:30 resulted in significantly lower demand than cloudy days, while demand increased during the light rain. Therefore, the weather should be considered in affecting car sharing services. We consider main factors of weather, including temperature (measured by ${ }^{\circ} \mathrm{C}$ ), and wind speed (measured by kilometers per hour), visibility (measured by kilometers), and condition which includes sunny (1), cloudy (2), light rain (3), light snow (4), moderate snow (5), moderate rain (6), heavy rain (7), heavy snow (8), and snowstorm (9). In this paper, the temperature, wind speed, visibility and condition during the $t$-th time interval are respectively denoted as $n t_{s}$, $n w_{s}, n v_{s}$, and $n c_{s}$.

4.2. LSTM. The long short-term memory artificial neural network (or LSTM) is a time recurrent neural network designed to solve long-term problems. All RNNs have a chained form of repetitive neural network modules. In a standard RNN, this repeated structural module has only a very simple structure, such as a tanh layer. LSTM introduces 


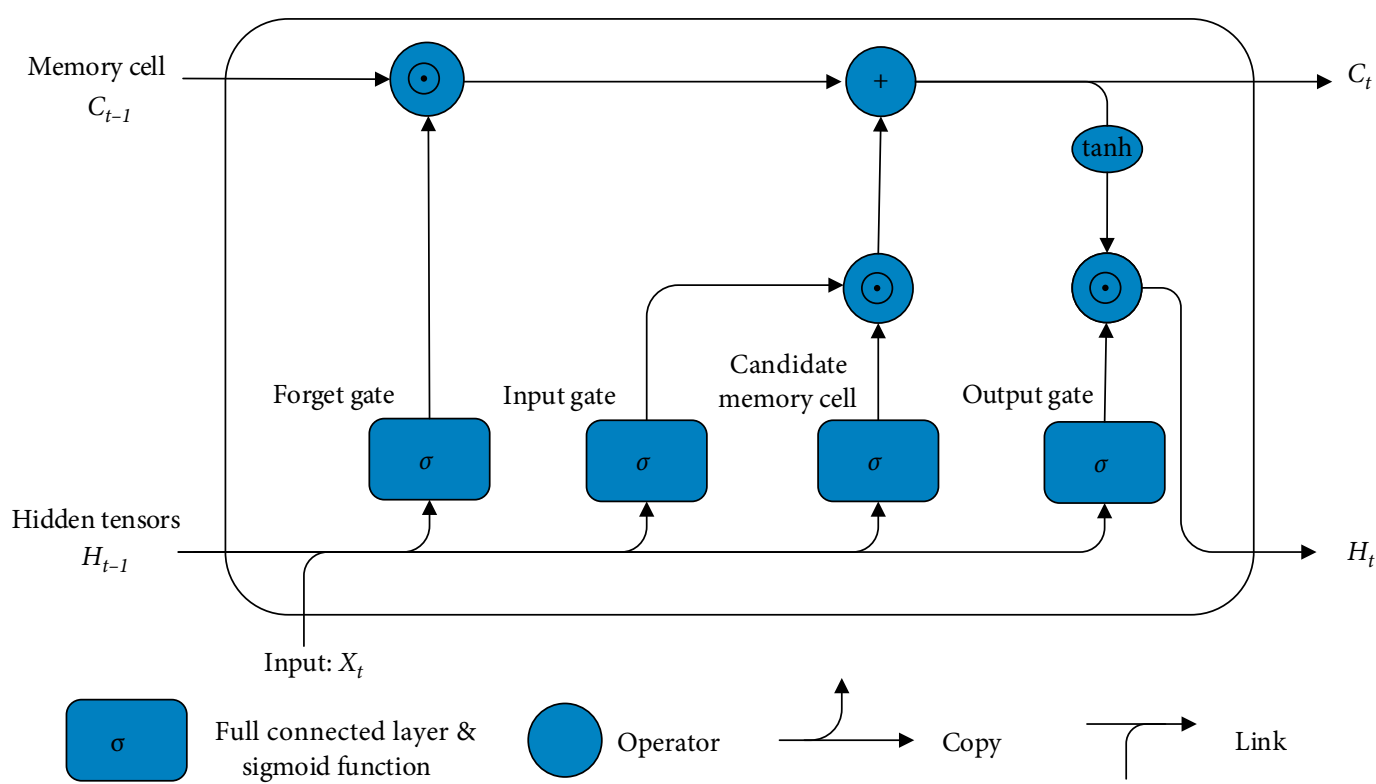

Figure 4: The inner structure of an LSTM layer.

three gates: input gate, forget gate, and output gate, as well as memory cells of the same shape as the hidden state, to record additional information. The reset gate and the update gate in the same door empty cycle unit are the same. As shown in Figure 4, the input gate of LSTM is the current time step input $\mathbf{X}_{t}$ and the previous time step hidden state $H_{t-1}$, and the output is calculated by the fully connected layer of the Sigmoid function. As a result, the value of the three gate elements is $[0,1]$. Specifically, assuming that the number of hidden cells is $h$, the small-batch input $\mathbf{X}_{t} \in R^{n \times d}$ (the number of samples is $n$, the number of inputs is $d$ ) and the previous time step hidden state $H_{t-1} \in R^{n \times h}$ is given for the time step $t$. The input gate $I_{t} \in R^{n \times h}$, the forgetting gate $F_{t} \in R^{n \times h}$ and the input gate $O_{t} \in R^{n \times h}$ of the time step are calculated as follows:

$$
\begin{gathered}
I_{t}=\sigma\left(\mathbf{X}_{t} \mathbf{W}_{x i}+H_{t-1} \mathbf{W}_{h i}+b_{i}\right), \\
F_{t}=\sigma\left(\mathbf{X}_{t} \mathbf{W}_{x f}+H_{t-1} \mathbf{W}_{h f}+b_{f}\right), \\
O_{t}=\sigma\left(\mathbf{X}_{t} \mathbf{W}_{x o}+H_{t-1} \mathbf{W}_{h o}+b_{o}\right) .
\end{gathered}
$$

LSTM needs to calculate a candidate memory cell $\hat{C}_{t}$, which is similar to the three gates, but the tanh function of the range $[-1,1]$ is used as the activation function. Specifically, the candidate memory cell $\hat{C}_{t} \in R^{n \times h}$ of a time step $t$ is calculated as

$$
\hat{C}_{t}=\tanh \left(\mathbf{X}_{t} \mathbf{W}_{x c}+H_{t-1} \mathbf{W}_{h c}+b_{c}\right),
$$

$\mathbf{W}_{x i}, \mathbf{W}_{x f}, \mathbf{W}_{x o} \in R^{d \times h}, \mathbf{W}_{h i}, \mathbf{W}_{h f}, \mathbf{W}_{h o} \in R^{h \times h}, \mathbf{W}_{x c} \in R^{d \times h}$, and $\mathbf{W}_{h c} \in R^{h \times h}$ are the weighted parameter matrices, while $b_{i}, b_{f}, b_{o} \in R^{1 \times h}$, and $b_{c} \in R^{1 \times h}$ are the intercept parameters.

The calculation of the current time step memory cell $C_{t}$ combines the information of the last time step and the current time step candidate memory cell, and controls the flow of information through the forget gate and the input gate (see Figure 4), using element multiplication (symbol as $\odot$ ),

$$
C_{t}=F_{t} \odot C_{t-1}+I_{t} \odot \hat{C}_{t} .
$$

Controlling the flow of information from memory cells to hidden state $H_{t} \in R^{n \times h}$ through the output gate

$$
H_{t}=O_{t} \odot \tanh \left(C_{t}\right) .
$$

The tanh function ensures that the hidden state element value is between -1 and 1 . When the output gate is approximately 1 , the memory cell information is passed to the hidden state for use by the output layer. When the output gate is approximately 0 , the memory cell information is retained by itself. Figure 4 shows the calculation of the hidden state in LSTM.

4.3. Structure for Time-Series Terms. Arbitrary time (including time-of-day and day-of-week) and weather variables (temperature, wind speed, visibility, and weather condition) are the other two classes of time-series variables. And separate architectures will be used, where each variable corresponds to a set of independent stacked LSTM layers. The stacked LSTM layers $L_{u}$ and $L_{v}$ are for number of vehicle pick-ups and dropoffs. Two high-level components $\hat{X}_{t}^{a}$ and $\hat{X}_{t}^{e}$ can be calculated and used for iteration in the above structure, respectively as

$$
\begin{gathered}
\left(A_{t-K_{u}+1}^{\left(L_{u}\right)}, \ldots, A_{t-1}^{\left(L_{u}\right)}\right)=F_{L_{u}}^{L} \ldots F_{1}^{L}\left(u_{t-K_{v}}, u_{t-K_{v}+1}, \ldots, u_{t-1}\right), \\
\hat{X}_{t}^{a}=F^{T}\left(F^{R}\left(\sigma\left(\omega_{a} * A_{t-1}^{\left(L_{u}\right)}+b_{a}\right)\right)\right) \\
\left(E_{t-K_{v}+1}^{\left(L_{v}\right)}, \ldots, E_{t}^{\left(L_{v}\right)}\right)=F_{L_{v}}^{L} \ldots F_{1}^{L}\left(v_{t-K_{v}}, v_{t-K_{v}+1}, \ldots, v_{t-1}\right)
\end{gathered}
$$




$$
\hat{X}_{t}^{e}=F^{T}\left(F^{R}\left(\sigma\left(\omega_{e} * E_{t}^{\left(L_{v}\right)}+b_{e}\right)\right)\right)
$$

where $A_{t-k}^{\left(L_{u}\right)}\left(k=1,2, \ldots, K_{u}\right)$ and $E_{t-v}^{\left(L_{v}\right)}\left(k=1,2, \ldots, K_{v}\right)$ are the output hidden tensors in the highest-level layers of the architectures of vehicle pick-ups and drop-offs, respectively. $K_{a}$ and $K_{e}$ are the look-back time windows. $\omega_{a}$ and $\omega_{e}$ are the weights, while $b_{u}$ and $b_{v}$ are the intercept parameters.

Out of respect for the influence of time and weather variables on demands, we define vectors $m_{s}=\left(f_{s}, y_{s}\right)$ and $n_{s}=\left(n t_{s}, n w_{s}, n v_{s}, n c_{s}\right)$, where $s=1,2, \ldots, t$, and feed them into the stacked LSTM architecture, producing the two highlevel components $\hat{X}_{t}^{p}$ and

$$
\begin{gathered}
\left(p_{t-K_{m}+1}^{\left(L_{m}\right)}, \ldots, p_{t}^{\left(L_{m}\right)}\right)=F_{L_{m}}^{L} \ldots F_{1}^{L}\left(m_{t-K_{m}+1}, \ldots, m_{t-1}, m_{t}\right) \\
\hat{X}_{t}^{p}=F^{T}\left(F^{R}\left(\sigma\left(\omega_{p} * p_{t-1}^{\left(L_{p}\right)}+b_{p}\right)\right)\right) \\
\left(q_{t-K_{n}+1}^{\left(L_{n}\right)}, \ldots, q_{t-1}^{\left(L_{n}\right)}\right)=F_{L_{n}}^{L} \ldots F_{1}^{L}\left(n_{t-K_{n}}, n_{t-K_{n}+1}, \ldots, n_{t-1}\right) \\
\hat{X}_{t}^{q}=F^{T}\left(F^{R}\left(\sigma\left(\omega_{q} * q_{t-1}^{\left(L_{q}\right)}+b_{q}\right)\right)\right)
\end{gathered}
$$

where $p_{t-k}^{\left(L_{m}\right)}\left(k=1,2, \ldots, K_{m}\right)$ and $q_{t-k}^{\left(L_{n}\right)}\left(k=1,2, \ldots, K_{n}\right)$ are the output hidden tensors in the highest LSTM layers $L_{m}$ and $L_{n} . K_{m}$ and $K_{n}$ are the look-back time windows of time and weather variable, respectively, while $L_{m}$ and $L_{n}$ are the number of designed LSTM layers for time and weather variable. $\omega_{p}$ and $\omega_{q}$ are the weights, while $b_{p}$ and $b_{q}$ are the intercept parameters.

4.4. Fusion. Considering incorporation of the diverse influences of high-level components, Hadamard product "o" will be employed to yield these components by multiplication with their respective parameter matrices $W_{a}, W_{e}, W_{p}$, and $W_{q}$, which is given by:

$$
\hat{X}_{t}=W_{a} \circ \hat{X}_{t}^{a}+W_{e} \circ \hat{X}_{t}^{e}+W_{p} \circ \hat{X}_{t}^{p}+W_{q} \circ \hat{X}_{t}^{q}
$$

Minimize the gap between the forecasted and the real-world demand, given by the squared difference of them, to learn the weighted and intercept parameters, during the training process. And to avoid overfitting, an L2-norm regularization term is included. And the objective function can be given by

$$
\min _{w, b}\left\|X_{t}-\hat{X}_{t}\right\|_{2}^{2}+\alpha\|W\|_{2}^{2} \text {, }
$$

where $\hat{X}_{t}$ expresses the real demand intensity, $\alpha$ is the regularization parameter set to balance the bias-variance trade-off, and $W$ presents all the weighted parameters in $X_{t}$. The training of the LSTM can be illustrated in Algorithm 1.
Input Record on number of pick-ups $\left\{u_{1}, \ldots, u_{i}, \ldots, u_{n}\right\}$ in training dataset

Record on number of drop-offs $\left\{v_{1}, \ldots, v_{i}, \ldots, v_{n}\right\}$ in training dataset

Record on time-of-day $\left\{f_{1}, \ldots, f_{i}, \ldots, f_{n}\right\}$

Record on day of week $\left\{y_{1}, \ldots, y_{i}, \ldots, y_{n}\right\}$ in training dataset

Record on number of weather variables $\left\{n t_{1}, \ldots, n t_{i}, \ldots, n t_{n}\right\}, \quad\left\{n w_{1}, \ldots, n w_{i}, \ldots, n w_{n}\right\}$, $\left\{n v_{1}, \ldots, n v_{i}, \ldots, n v_{n}\right\},\left\{n c_{1}, \ldots, n c_{i}, \ldots, n c_{n}\right\}$

Look-back windows $K_{a}, K_{e}, K_{m}$ and $K_{n}$

Output LSTM with learnt parameters

\section{1: Procedure LSTM training}

2: $\quad$ initialize a null set: $S \leftarrow \varnothing$

3: for all defined time slice $t$ do

4: $\quad \pi_{t}^{a} \leftarrow\left[u_{t-K_{a}}, u_{t-K_{a}+1}, \ldots, u_{t-1}\right]$

5: $\quad \pi_{t}^{e} \leftarrow\left[v_{t-K_{e}}, v_{t-K_{e}+1}, \ldots, v_{t-1}\right]$

6: $\quad \pi_{t}^{m} \leftarrow\left[m_{t-K_{m}}, \ldots, m_{t-1}, m_{t}\right]$, where $m_{s}=\left(f_{s}, y_{s}\right)$

7: $\quad \pi_{t}^{n} \leftarrow\left[n_{t-K_{n}}, \ldots, n_{t-1}, n_{t}\right]$, where $n_{s}=\left(n t_{s}, n w_{s}, n v_{s}, n c_{s}\right) \triangleright$, where $\pi_{t}^{a}, \pi_{t}^{e}, \pi_{t}^{p}, \pi_{t}^{q}$ are the sets of different categories of explanatory variables in one observation

8: $\quad$ A training observation $\left(\pi_{t}^{a}, \pi_{t}^{e}, \pi_{t}^{p}, \pi_{t}^{q}, A_{t}\right)$ is put into $L$

9: A training observation $\left(\pi_{t}^{a}, \pi_{t}^{e}, \pi_{t}^{p}, \pi_{t}^{q}, E_{t}\right)$ is put into $L$

\section{0: end for}

11: initialize all the weighted and intercept parameters

12: repeat

13: $\quad$ randomly extract a batch of samples $L^{b}$ from $L$

14: estimate the parameters by the minimization of the objective function shown in Equation (30) within $L^{b}$

15: until convergence criterion met

16: end procedure

\section{Algorithm 1}

5. An Experimental Case. This paper uses the data from two major station-based car sharing operators in Chengdu, $E$ and $G$, for instance verification. Both focus on the mobile platforms. $E$ features 24 h user self-service vehicle sharing service. It mainly provides long- and short-term new energy vehicle rental services to group users (B2B, B2B2C) and private users (B2C). $G$ is a new energy car sharing project that provides users with convenient, green, intelligent, economic, and fashionable means of urban transport.

5.1. Operation Background. The operating stations of both systems are marked on the map of Chengdu, as in Figure 5. The red triangles mark the stations in the car sharing system $G$, while the yellow filled circles mark the stations in the system $E$. The car sharing stations of system $E$ is dispersedly distributed and covers the city, while the stations of $G$ are 


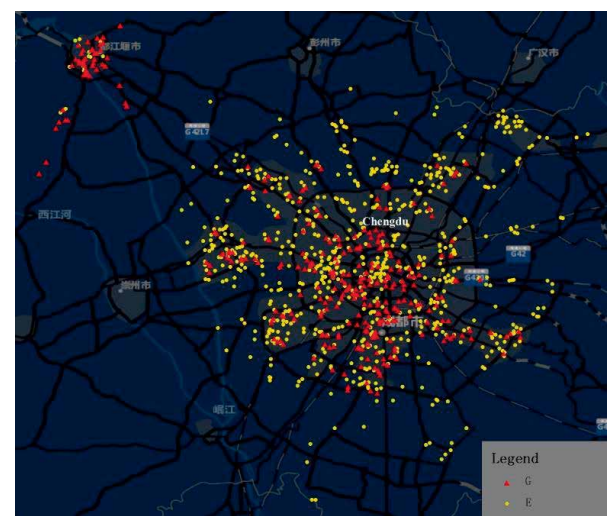

FIgURE 5: Car sharing station distribution for $G \& E$.

mainly located in the central zone of the city and area around Dujiangyan in the northwest outskirt of Chengdu.

As shown in Figures 6 and 7, the average hourly demands reflect the size of their business operations, where the hourly average demands on system $E$ are smaller than that on $G$. Do notice that the scales on the horizontal axes of the charts are different. The demand patterns during weekday and weekend are the same for system $E$, and so is true for system $G$. The hourly characteristics of the two systems can be easily differentiated. The first two peaks at around $10 \mathrm{am}$, and $5 \mathrm{pm}$ on the poly-line of system $G$ adhere to the traffic characteristics during the day, followed by leisure activities after work. However, the average hourly demands on system $E$ cannot be easily concluded in a pattern, which is likely the result of its pricing strategy as the fourth peak of system $G$.

We intend to present the attractiveness of the service in different driving times in the car sharing market. The attractiveness reflects the market's reaction towards certain strategies of the service providers, such as price rate per time used and other promotions. Note that driving time represents the utilization of operating vehicles to fulfil the intended transport demand of the customers $[38,39]$. Thus, it is possible to associate the driving time during a vehicle usage with usages to show the attractiveness of car sharing service over different driving times.

We deem the driving times whose differences from their average smaller than $\delta$-granularity be no different. In an urban traffic environment, the value of $\delta$ between two exact same stations is dependent on the traffic condition when the vehicle is used, given no significant differences between routing. Then, we have the set of driving times whose deviations are no larger than $\delta$ and express it as

$$
s\left(\bar{d}_{v}, \delta\right)=\left\{\bar{d}_{v}\right\},
$$

where $\bar{d}_{v}$ signs the average number of vehicles whose driving times are within $\left(\bar{d}_{v}-\delta, \bar{d}_{v}+\delta\right)$. And the number of e-cars whose driving times are $\bar{d}_{v}$ at $\delta$-granularity can be calculated by

$$
c\left(\bar{d}_{v}\right)=\sum_{d_{v} \in S\left(\bar{d}_{v} \delta\right)}\left|\frac{d_{v}}{d_{v}}\right| .
$$

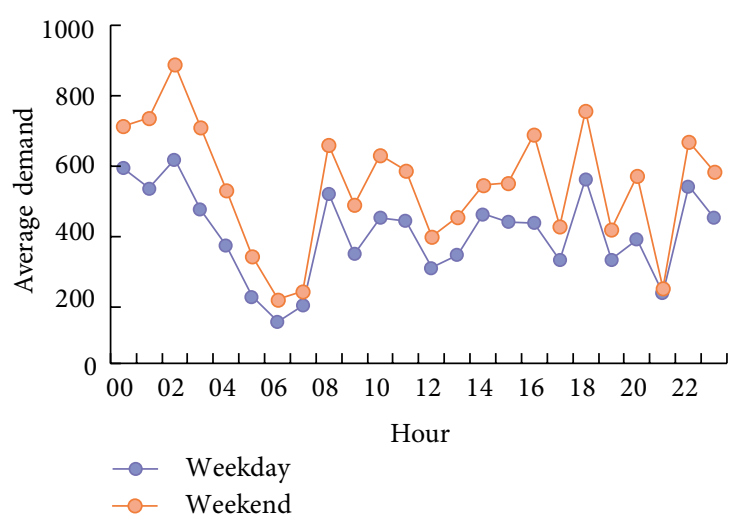

FIgURE 6: Hourly demand for $E$.

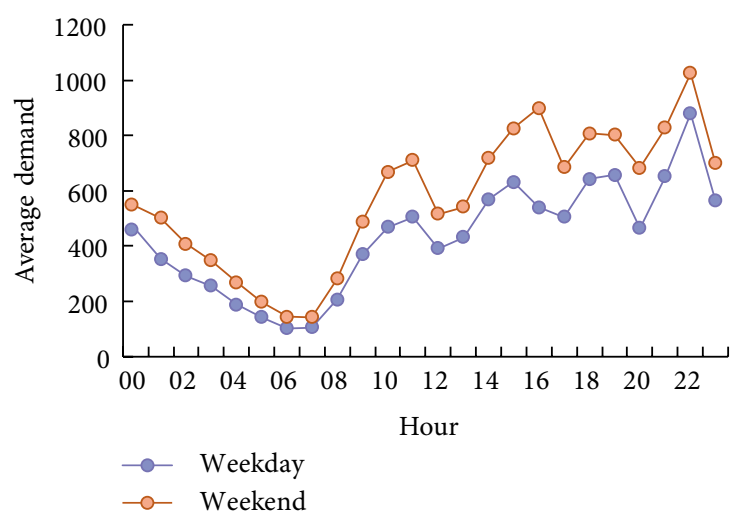

Figure 7: Hourly demand for G.

Suppose fitting function $f: \mathbb{Z}^{+} \rightarrow \mathbb{R}^{+}$exists, and its associated domain can be included in the managerial regulations. We exclude the scenario when $\bar{d}_{v}=0$ which denotes immediate pick-up and drop-off, and thus the domain of the fitting function is $\bar{d}_{v}>0$. The codomain of the function can be deemed the ratio of demands $c\left(\bar{d}_{v}\right)$ whose average driving time is $\bar{d}_{v}$ within $\delta$-granularity and the total demands $C$, namely $c\left(\bar{d}_{v}\right) / C$.

We exclude the driving time longer than $1600 \mathrm{~min}$ based on our review of operation data. Both the attractiveness of car sharing service from $G$ and $E$ can be fitted as a kernel density function using MATLAB, as shown in Figure 8.

As can be seen from the fitting curve, the vehicle uses of $E$ are almost 0 when the driving time exceeds $850 \mathrm{~min}$, and the uses between 850 and 1600 are rather scarce. The vehicle uses of $G$ becomes 0 at around $1500 \mathrm{~min}$ of driving time. And the vehicle uses of $G$ are not so concentrated during certain sections in comparison to that of $E$. The service of $E$ is considerably more attractive than that of $G$ when the driving time is between $25 \mathrm{~min}$ and $125 \mathrm{~min}$. In the meantime, the service of $G$ is more attractive than $E$ when driving time exceeds 125 , which could be the result of pricing strategy that service provider $G$ charges considerably less after a certain time of usage.

5.2. Data Preparation. Considering the general traffic condition and the average hourly demands on system $G$, the time periods during a day are defined as: 


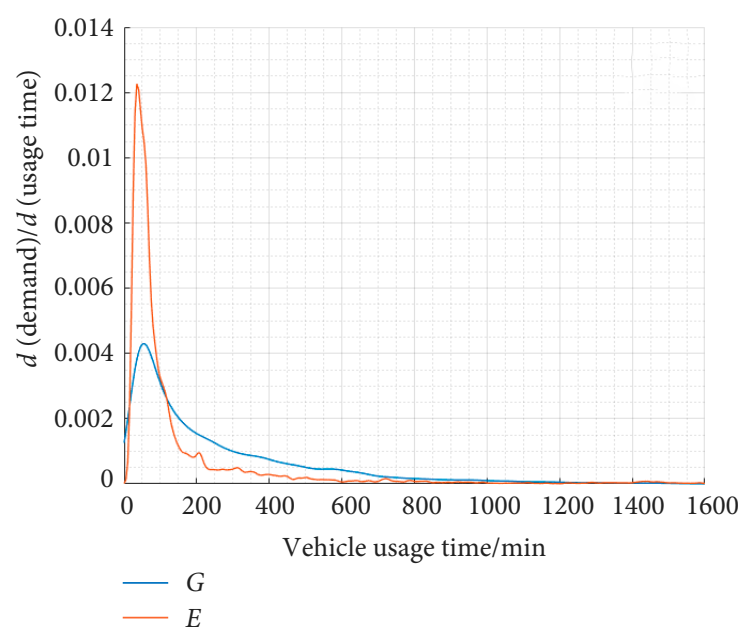

FIGURE 8: Kernel function fitting curve for $E \& G$.

(1) inactive period: 00:00-07:00;

(2) morning peak: 07:00-09:00;

(3) between morning and evening peak: 09:00-17:00;

(4) evening peak: 17:00-20:00;

(5) after evening peak: 20:00-24:00.

The relative frequency of vehicle utilization is immediately related to the attractiveness of car sharing to its consumers. The utilization of car sharing services from both companies share a consensus that most consumers are prone to using the service if the destination can be reached within 30-90 min of driving. This is especially so for $E$ company, whose relative frequency of demand within 30-90 is only slightly less than half of the total trips using its service (see Figure 9). Besides, there are noteworthy differences between $E$ and $G$ in terms of average driving time as well as average driving distance. The average driving time of $E$ is $166 \mathrm{~min}$, while that of $G$ is $267 \mathrm{~min}$, slightly less than that of $E$.

Main users use the scar sharing service of $G$ within the driving distance of $G$ from $5 \mathrm{~km}$ to $20 \mathrm{~km}$, while most driving distances of $E$ range from $2 \mathrm{~km}$ to $15 \mathrm{~km}$ (see Figure 10). The average driving distance of trips from system $E$ is $11.39 \mathrm{~km}$, while that from $G$ is $6.85 \mathrm{~km}$, which is slightly less than that of $E$.

5.3. Demand Prediction. The interval of a dataset for temporal series variables is set to be one hour. We recognize that a single trip demand on the car sharing system originates from one of its stations. We visualize the data for prediction in such form: pick-up is represented in red pillars and drop-off in blue, and their heights are proportionate to the numbers of pick-ups (drop-offs). The LSTM is trained on the training dataset and validated on the test dataset, respectively. In the same vein, the LSTM network is only fed with the historical demand dataset.

We use LSTM as the unconditional short-term prediction model, which includes all the variables, number of vehicles at the station, usage distribution, time of day, and day of week. The lookback time window is set to be $K=4$. And the
TABLE 1: Performance comparison of different approaches.

\begin{tabular}{lccc}
\hline Model & RMSE & $R^{2}$ & MAE \\
\hline ANN & 0.0375 & 0.812 & 0.0205 \\
CNN & 0.0319 & 0.803 & 0.0189 \\
LSTM & 0.0298 & 0.794 & 0.0182 \\
\hline
\end{tabular}

TABLE 2: GPS data catalogue.

\begin{tabular}{lc}
\hline Data types & Data content \\
\hline Routine & Pick-up time \\
Routine & Drop-off time \\
Longitude and latitude & Car starting coordinates \\
Longitude and latitude & Car destination coordinates \\
\hline
\end{tabular}

predicted term is vehicle usages at all car sharing stations on a natural day.

In LSTM, we include three variants of our model with a different number of layers. The model utilizes historical data: numbers of vehicle pick-up/drop-off, arbitrary time, and weather variables, to predict future vehicle pick-ups and dropoffs. The structuring of LSTM is introduced in Section 4.2.

We evaluate the model by three effectiveness measures (root mean squared error (RMSE), coefficient of determination $R^{2}$, mean absolute error (MAE)) to measure the total prediction accuracy/degree fitting in the whole test data [32]. Table 1 shows that our proposed model is statistically significant in all three effectiveness measures. It can be found that the proposed LSTM is superior to the benchmarks in the measurement of predicting performance. LSTM achieves the best predictive performance measured by RMSE (0.0298), which is $6.5 \%$ lower than $\mathrm{CNN}$ (0.0319). In car sharing system, $\mathrm{CNN}$ captures spatial correlation and is superior to $\mathrm{ANN}$, which only considers temporal features. It is indispensable to include spatial correlation for predicting station-based car sharing demands.

Figure 11 presents the heat maps showing the basic condition of vehicle distribution during respective periods, where deeper color denotes larger demand intensity. The scattered utilization of shared vehicles during morning commuting peaks and the correspondingly concentrated utilization during evening commuting peaks demonstrated on the predicted results coincides with urban traffic conditions. In the meantime, vehicle usages in noncommuting periods are mainly distributed in the city center and tourist sites. It is proven to be true that different urban traffic patterns will lead to different distribution characteristics of station-based car sharing system.

According to the output of LSTM, we obtained a one-day predicted OD data of $E$ and $G$ with 4989 records in total, and the data catalogue is shown in Table 2.

In the above 16 three-dimensional maps, red represents $E$, and blue represents $G$. The columnar height of the site represents the strength of the demand. Figure 12 is a screenshot of the pick-up. From an overview, we find that the demands for car sharing service in Chengdu are remarkably consistent with the trip habits of Chengdu residents. As Chengdu is 

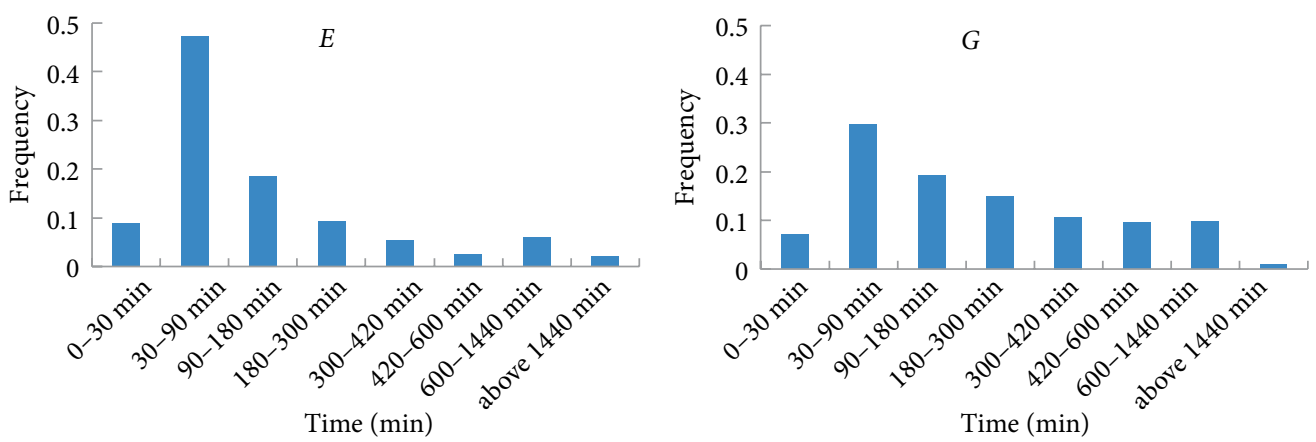

FIgURE 9: Driving time distribution.
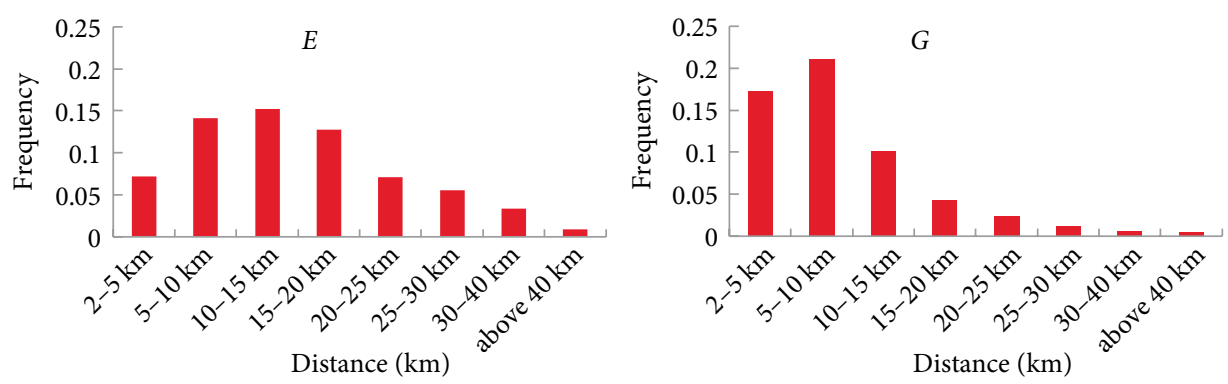

FIGURE 10: Driving distance distribution.

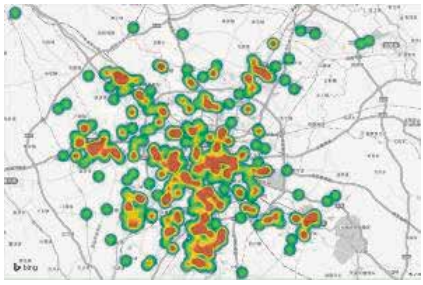

(a)

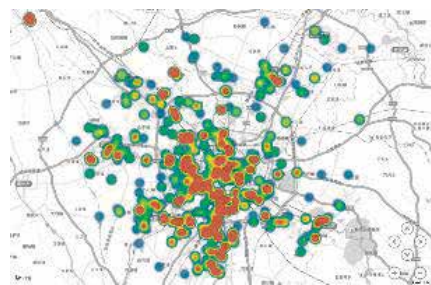

(d)

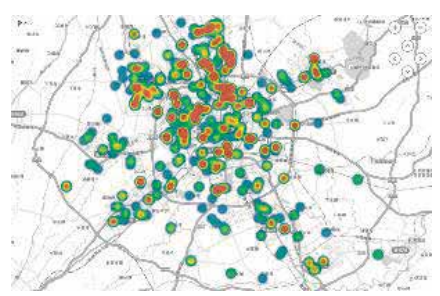

(b)

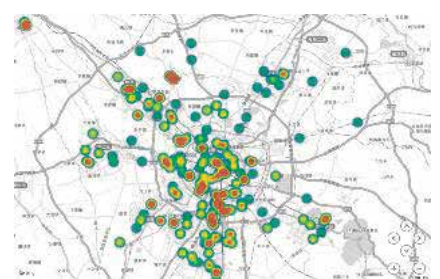

(e)

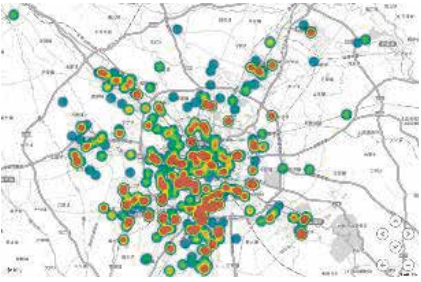

(c)

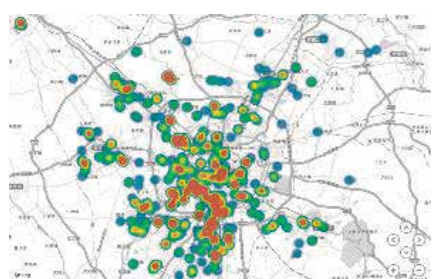

(f)

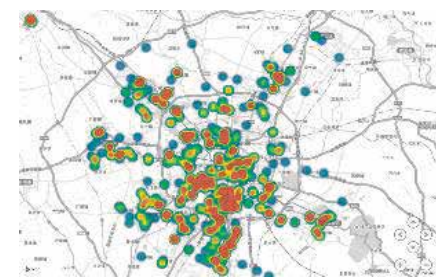

(g)

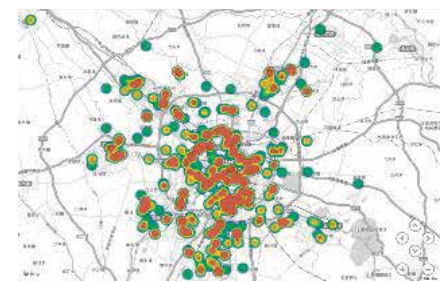

(h)

Figure 11: Real demand distribution on the map during different time periods. (a) 00:00-07:00. (b) 07:00-09:00. (c) 09:00-12:00. (d) 12:0014:00. (e) 14:00-17:00. (f) 17:00-19:00. (g) 19:00-22:00. (h) 22:00-24:00.

economically developing its southern region, as well as commerce agglomerating there, the demands in the inactive period are significantly higher than in other regions. During the morning peak period, the demands for car sharing service in the entire system may not be high due to traffic jams in the metropolis. At noon, the demands are transferred to the most 


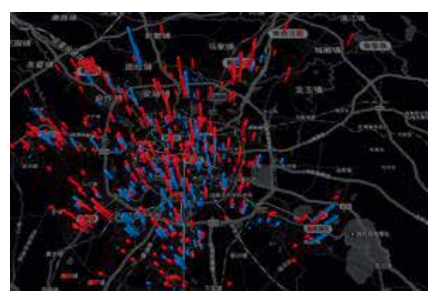

(a)

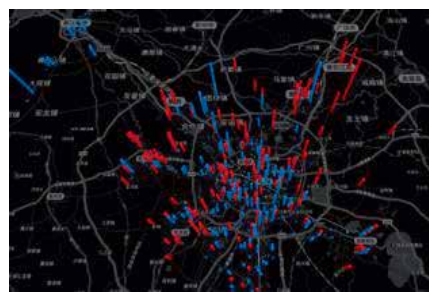

(d)

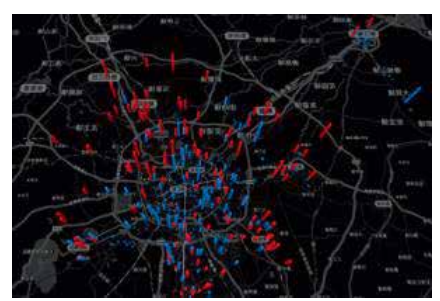

(b)

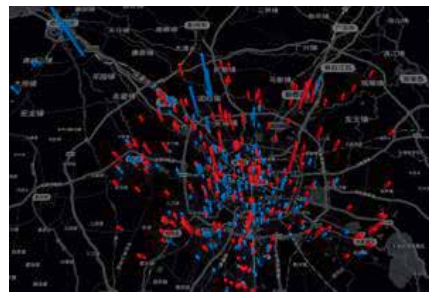

(e)

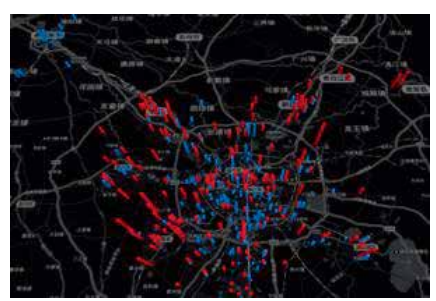

(c)

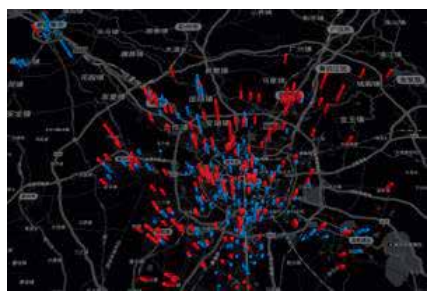

(f)

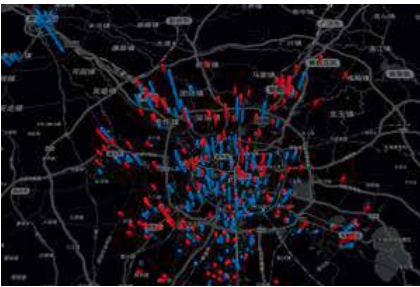

(g)

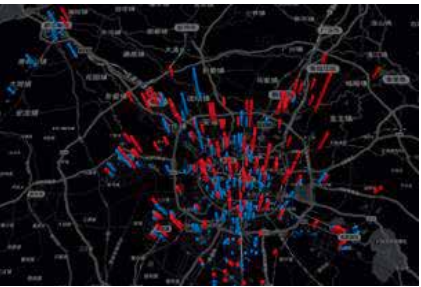

(h)

Figure 12: Predicted pick-up demand distribution on map during different time periods. (a) 00:00-07:00. (b) 07:00-09:00. (c) 09:00-12:00. (d) 12:00-14:00. (e) 14:00-17:00. (f) 17:00-19:00. (g) 19:00-22:00. (h) 22:00-24:00.

CBDs in Chengdu. The demands during the afternoon and the evening peak is not that high. But what's interesting is that Chengdu, as an attractive tourist city, generates the highest demands during the day. After the evening peak, the demand is less balanced than between morning and evening peak due to the regulation placed on driving. Predicted results demonstrate that the utilization of deep learning forecasting techniques based on given initial resource distribution as well as visualization can help car sharing operators and government to detect changes of demand distribution, based on which a more well-based adjusting strategy can be designed to avoid these imbalanced conditions. As far as the two companies are concerned, the number of vehicles and site settings invested by $E$ is much higher than that of $G$. Therefore, from this visualization, it is not possible to determine which operator's operating mode is better.

Figure 13 is a three-dimensional visual view of drop-off. The situation shown in the figure is just the opposite of the pick-up. From morning to afternoon, the distribution and intensity of drop-off have been stabilizing. After $5 \mathrm{pm}$, the drop-off intensity in the city center suddenly increased sharply and continued until the end of the evening. The visualization results in Figures 12 and 13 tell us that the OD data learned by the LSTM structure is in line with the situation in Chengdu, which can bring the most reliable guarantee for the management research of further car sharing systems.

5.4. Analysis of Predicted Results: Spontaneous Deviation. Using the predicted data, the matrix demonstrating the spontaneous deviations that the operation imposes on the system can be calculated, as well as the indicators for showing the operating condition of the car sharing system. For normal operation of the car sharing system without vehicle malfunctioning, the vehicle leased out of the system should always return to the same system, meaning

$$
\sum_{i} \sum_{j} z_{i}^{t_{j}, t_{j+1}}=0,
$$

where $\left(t_{j}, t_{j+1}\right)$ covers one-day operation. However, it is not always so in one day's operation. Some users may prefer to use car sharing services overnight, which may cause a deviation between pick-up and drop-off within one day.

The overall deviations, from the perfect condition of operation, of company $E$ and $G$ are calculated as $I_{E}=-13$, and $I_{G}=47$. The operational interpretation of $I_{E}$ being negative means that up to the end of the predicted operation day, more vehicles were borrowed than returned. And the operational interpretation of $I_{G}$ being positive 47 means that up to the end of the predicted operation day, more vehicles were returned than borrowed, compared with $I_{E}$. And it can be predicted based on Equation (33) that negative and positive values should alternately appear, when predicting more than one day of operation.

Concerning the operational status of the two companies, their standardized variances of deviation are calculated as $D_{E}=0.671$, and $D_{G}=2.005$. It can be easily interpreted that the performance on resource distribution and management of service provider $E$ is better than that of provider $G$ since they are the accumulated value of the deviations in system 


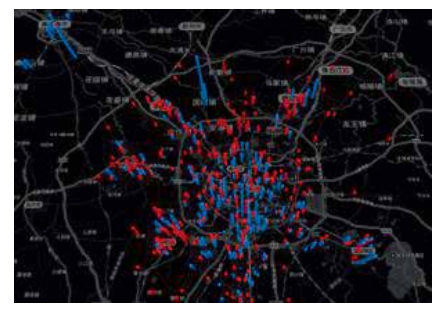

(a)

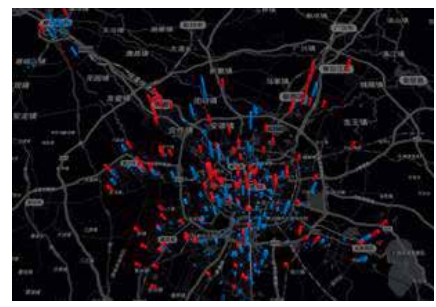

(d)

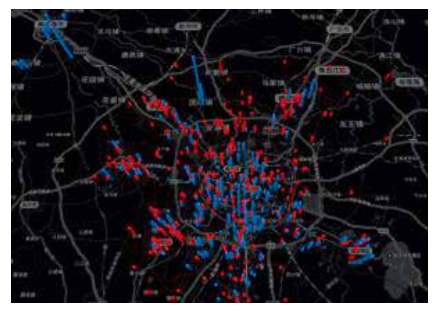

(b)

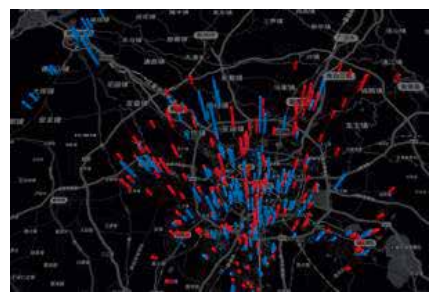

(e)

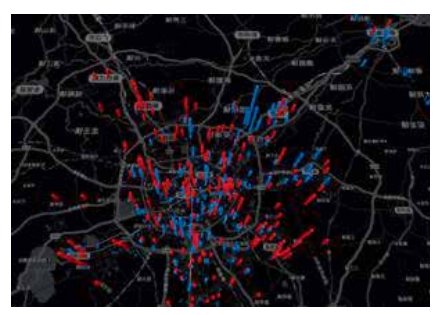

(c)

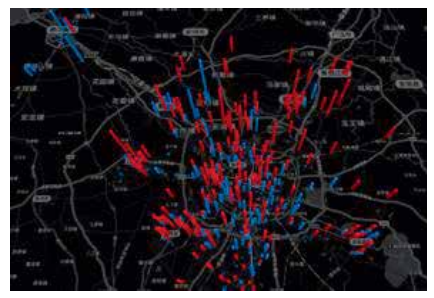

(f)

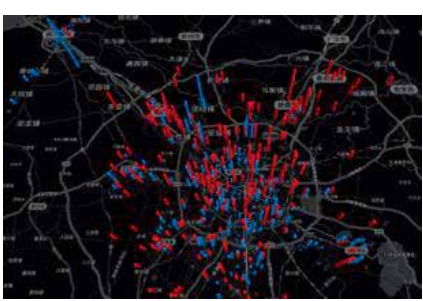

(g)

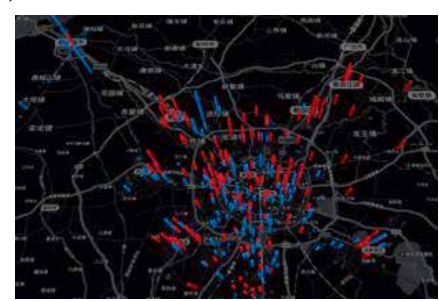

(h)

FiguRE 13: Predicted drop-off demand distribution on map during different time periods. (a) 00:00-07:00. (b) 07:00-09:00. (c) 09:00-12:00. (d) 12:00-14:00. (e) 14:00-17:00. (f) 17:00-19:00. (g) 19:00-22:00. (h) 22:00-24:00.

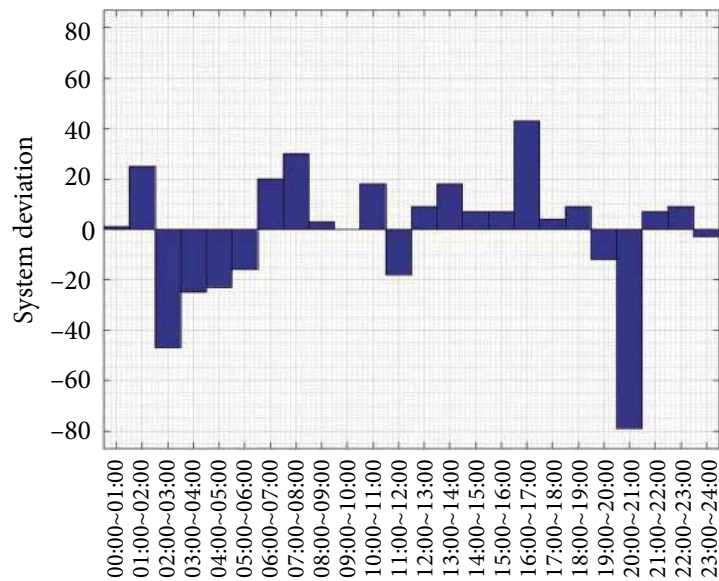

(a)

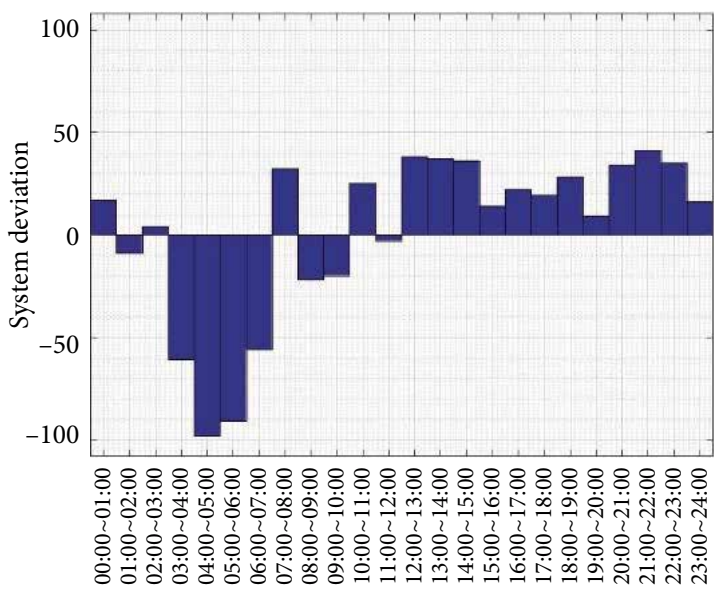

(b)

Figure 14: The hourly deviation of system $E \& G$.

management from real market demands. Based on Figure 14, a noticeable difference can already be told from the hourly performance of the whole system. Even though the deviation of system $E$ in 20:00-21:00 is quite large, but not as that of $G$. Deviations of system $G$ in 03:00-04:00 and 04:00-05:00 are both above 90 , while others in the meantime are greater than that of system $E$. The station-wise daily deviations of $E$ are distinguishably better gentle than that of $G$ based on Figure 15 , not to mention that provider $E$ has more than twice stations than $G$. In a nutshell, the operating condition of $E$ car sharing system is more favourable than that of $G$.

\section{Conclusion}

Car sharing service is easy and efficient to use due to a pre-verification process, while electricity-powered vehicles are commonly regarded environment-friendly. It makes electric car sharing especially appealing to large cities, such as Chengdu, that must process transport demands efficiently with relatively environment-friendly means of transport. Even though the concept of car sharing is no longer new, there still lacks a general understanding of the emerging features of its operation, such as the spontaneous deviating process caused by vehicle 


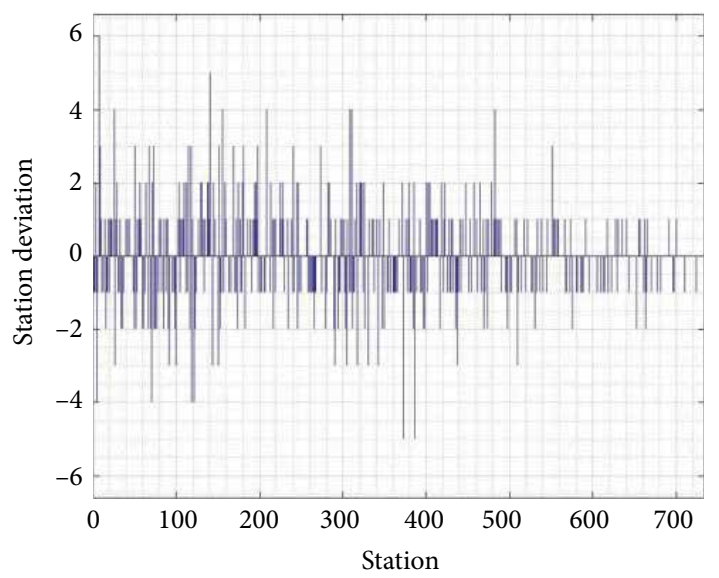

(a)

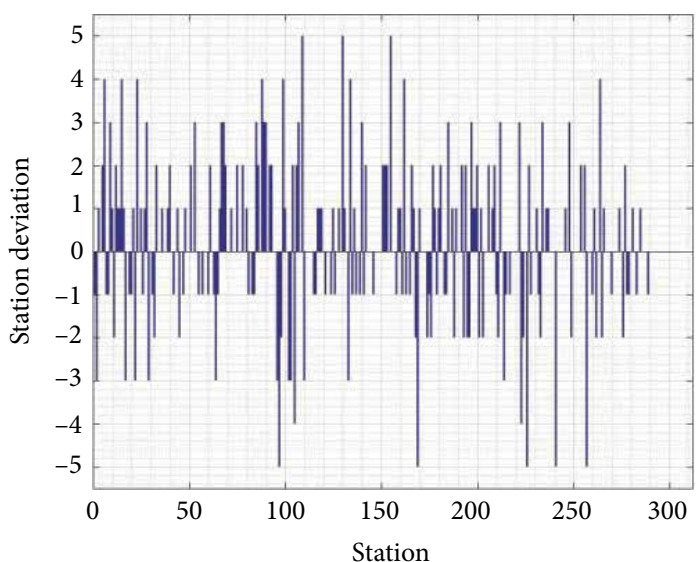

(b)

Figure 15: The daily deviation of station $i$ for $E \& G$.

utilization behaviours within the system. This paper seeks to detect the operation status of car sharing systems.

And the system evaluation is based on demand forecasting using deep learning techniques. Through establishing an LSTM structure for forecasting vehicle pick-up and drop-off over time, we consider multiple temporal features, including the time of day, day of week, and weather condition. Based on the predicted data, the demand pattern of station-based car sharing can be predicted in alignment with urban traffic, which shows that model formulation of pick-up and drop-off is effective for short-term demand prediction for station-based car sharing services. And the operation of a station-based car sharing system can thus be better understood with the help of the LSTM structure.

This paper includes a discrete event model, which enables the interdependency analysis of car sharing demands with their temporal indications and calculates the deviation matrix representing the balances of pick-ups and drop-offs at different times (though not presented in the paper), which demonstrates the deviation of vehicle distribution from vehicle demand. The results showed that the analytical system is feasible in terms of depicting the system status in combination with LSTM forecasting, which can be used as a baseline for further adapting strategies of the system.

This paper also analyses the correlation of vehicle usages with driving times, which helps identify the market segment of station-based car sharing (among the various means of urban transport) and demonstrates the reaction of the car sharing market towards the operating strategies based on the data from two service providers. We presume that it is responsive to managerial interventions that are important for improving car sharing operation, including operator relocation or pricing, to intervene in the redistribution process. Therefore, a potential research opportunity lies in predicting the market's reaction to operation strategies based on the proposed analytical system combined with deep learning techniques, helping decision-makers optimize their operation strategies to achieve the optimal overall state of operation and to acquire maximum profit.

\section{Data Availability}

Access to data is restricted. The survey data source has certain confidentiality.

\section{Conflicts of Interest}

The authors declare that they have no conflicts of interest.

\section{Funding}

This research is supported by the National Science Foundation (NSF) of China under Grant No. U1733127.

\section{Acknowledgments}

The authors thank the two anonymous reviewers for their valuable comments.

\section{References}

[1] S. Shaheen, D. Sperling, and C. Wagner, "Carsharing in Europe and North America: past, present, and future," Transportation Quarterly, vol. 52, no. 3, pp. 35-52, 1998.

[2] Z. F. Yang, "The development characteristics, evolutionary path and promotion measures of the global sharing economy," International Trade, vol. 34, pp. 58-59, 2018.

[3] "The two ministries jointly issued the "guiding opinions on promoting the healthy development of small and mini bus leases"', 2018, https://m.huanqiu.com/article/9CaKrnK4H0u.

[4] "Ranking of urban motor vehicle possession," 2018, http://www. sohu.com/a/218260672_400718.

[5] Z. Y. Luo and H. Leng, "Analysis on the problems and impacts of the development of internet cars under the background of legalization," vol. 10, China Urban Planning Annual Meeting Hangzhou, Zhejiang, China, 2018.

[6] S. A. Shaheen and A. P. Cohen, "Carsharing and personal vehicle services: worldwide market developments and emerging 
trends," International Journal of Sustainable Transportation, vol. 7, no. 1, pp. 5-34, 2012.

[7] "China internet car timeshare leasing market special analysis 2017," Analysyis: China 2017.

[8] G. Brandstätter, M. Kahr, and M. Leitner, "Determining optimal locations for charging stations of electric car-sharing systems under stochastic demand," Transportation Research Part B: Methodological, vol. 104, pp. 17-35, 2017.

[9] S. A. Shaheen and A. P. Cohen, "Growth in worldwide carsharing," Transportation Research Record: Journal of the Transportation Research Board, vol. 1992, no. 1, pp. 81-89, 2007.

[10] Wikipedia: Carsharing, 2018, https://en.wikipedia.org/wiki/ Carsharing.

[11] The CarSharing Handbook (Part 1), 2007, Retrieved 17 April Rain Magazine:2019.

[12] "Five major development trends in the car sharing industry in 2019," 2019.

[13] "China car-sharing status and trend report 2017," Southern Daily, China, 2017.

[14] C. K. Orski, “Car sharing," Transportation Quarterly, vol. 55, pp. 13-15, 2001.

[15] F. E. Prettenthaler and K. W. Steininger, "From ownership to service use lifestyle: the potential of car sharing," Ecological Economics, vol. 28, no. 3, pp. 443-453, 1999.

[16] N. T. Fellows and D. E. Pitfield, "An economic and operational evaluation of urban car-sharing," Transportation Research Part D: Transport and Environment, vol. 5, no. 1, pp. 1-10, 2000.

[17] R. Katzev, "Car sharing: a new approach to urban transportation problems," Analyses of Social Issues and Public Policy, vol. 3, no. 1, pp. 65-86, 2003.

[18] J. Firnkorn and M. Müller, "What will be the environmental effects of new free-floating car-sharing systems? The case of car2go in Ulm," Ecological Economics, vol. 70, no. 8, pp. 1519-1528, 2011.

[19] M. Heilig, N. Mallig, O. Schröder, M. Kagerbauer, and P. Vortisch, "Implementation of free-floating and station-based carsharing in an agent-based travel demand model," Travel Behaviour and Society, vol. 12, pp. 151-158, 2018.

[20] H. Wang, R. Cheu, and D.-H. Lee, "Logistical inventory approach in forecasting and relocating share-use vehicles," in International Conference on Advanced Computer Control, IEEE, Shenyang, China, 2010.

[21] F. Ciari, N. Schuessler, and K. W. Axhausen, "Estimation of carsharing demand using an activity-based microsimulation approach: model discussion and some results," International Journal of Sustainable Transportation, vol. 7, no. 1, pp. 70-84, 2013.

[22] T. Yoon, C. R. Cherry, M. S. Ryerson, and J. E. Bell, "Carsharing demand estimation and fleet simulation with EV adoption," Journal of Cleaner Production, vol. 206, pp. 1051-1058, 2019.

[23] G. Alfian, J. Rhee, M. Ijaz, M. Syafrudin, and N. Fitriyani, "Performance analysis of a forecasting relocation model for one-way carsharing," Applied Sciences, vol. 7, no. 6, p. 598, 2017.

[24] A. Ghaderi, B. M. Sanandaji, and F. Ghaderi, "Deep forecast: deep learning-based spatio-temporal forecasting," ICML 2017 Time Series Workshop, Sydney, Australia, 2017.

[25] T. Epelbaum, F. Gamboa, J. Loubes, and J. Martin, "Deep learning applied to road traffic speed forecasting," 2017.

[26] J.-X. Xu and J. S. Lim, "A new evolutionary neural network for forecasting net flow of a car sharing system," in 2007 IEEE
Congress on Evolutionary Computation, pp. 1670-1676, IEEE, Singapore, 2007.

[27] H. L. Khoo, "An application of intelligent noise filtering techniques in demand forecasting for carsharing systems," International Journal of ITS Research, vol. 6, 2008.

[28] B. Yu, H. Yin, and Z. Zhu, "Spatio-temporal graph convolutional networks: a deep learning framework for traffic forecasting," 2017.

[29] W. Zhang, Y. Yu, Y. Qi, F. Shu, and Y. Wang, "Short-term traffic flow prediction based on spatio-temporal analysis and CNN deep learning," Transportmetrica A: Transport Science, vol. 15, no. 2, pp. 1688-1711, 2019.

[30] Y. Ai, Z. Li, M. Gan et al., "A deep learning approach on shortterm spatiotemporal distribution forecasting of dockless bikesharing system," Neural Computing and Applications, vol. 31, no. 5, pp. 1665-1677, 2019.

[31] L. Wang, Y. Zhong, and W. Ma, "GPS-data-driven dynamic destination prediction for on-demand one-way carsharing system," IET Intelligent Transport Systems, vol. 12, no. 10, pp. 1291-1299, 2018.

[32] J. Ke, H. Zheng, H. Yang, and X. (M.) Chen, "Short-term forecasting of passenger demand under on-demand ride services: a spatio-temporal deep learning approach," Transportation Research Part C: Emerging Technologies, vol. 85, pp. 591-608, 2017.

[33] Y.-L. Gu, M. Li, X-. P. Rui, W-.Q. Lu, and S. Wang, "Short-term forecasting of supply-demand gap under online car-hailing services based on deep learning," Journal of Transportation Systems Engineering and Information Technology, vol. 19, pp. 223-230, 2019.

[34] F. Rodrigues, I. Markou, and F. C. Pereira, "Combining timeseries and textual data for taxi demand prediction in event areas: a deep learning approach," Information Fusion, vol. 49, pp. 120-129, 2019.

[35] L. Shen and F. Tao, "Optimization of vehicle management for one-way carsharing systems based on time windows of users," in 8th International Conference on Industrial Technology and Management (ICITM), pp. 110-114, IEEE, Cambridge, UK, 2019.

[36] G. Molnar and G. H. de A. Correia, "Long-term vehicle reservations in one-way free-floating carsharing systems: a variable quality of service model," Transportation Research Part C: Emerging Technologies, vol. 98, pp. 298-322, 2019.

[37] G. S. Fishman, "Discrete-event simulation: modeling, programming, and analysis," Journal of the Royal Statistical Society, vol. 52, pp. 408-409, 2010.

[38] G. Linden, S. Hanks, and N. Lesh, "Interactive assessment of user preference models: the automated travel assistant," Proceeding International Conference on User Modeling, 1997.

[39] S. Y. Jung, J.-H. Hong, and T.-S. Kim, "A statistical model for user preference," IEEE Transactions on Knowledge \& Data Engineering, vol. 17, pp. 834-843, 2005. 